\title{
Clinical features of 64 patients (outside Hubei) with COVID-19 in Wenzhou, China
}

\author{
Zhangwei Qiu ${ }^{1 \#}$, Wei Dai ${ }^{2 \#}$, Madiha-Zahra Syeda ${ }^{3 \#}$, Liujian Ouyang ${ }^{3,4 \#}$, Jie Lin $^{1}$, Laifang Sun ${ }^{5}$, \\ Zhefeng Leng ${ }^{1}$, Xinmiao Chen ${ }^{1}$, Xiaoting Xu ${ }^{1}$, Yaxin Zhao ${ }^{6}$, Binyu Ying ${ }^{7}$, Xian Shen ${ }^{6}$, Songmin Ying ${ }^{3}$, \\ Yuanrong Dai ${ }^{1}$
}

\begin{abstract}
${ }^{1}$ Department of Respiratory and Critical Care Medicine, ${ }^{2}$ Department of Neurorehabilitation, The Second Affiliated Hospital and Yuying Children's Hospital of Wenzhou Medical University, Wenzhou, China; ${ }^{3}$ Department of Pharmacology \& Department of Respiratory and Critical Care Medicine of the Second Affiliated Hospital, Zhejiang University School of Medicine, Key Laboratory of Respiratory Disease of Zhejiang Province, Hangzhou, China; ${ }^{4}$ Chu Kochen Honors College of Zhejiang University, Hangzhou, China; ${ }^{5}$ Department of Emergency Medicine, ${ }^{6}$ Department of General Surgery, ${ }^{7}$ Department of Intensive Care Unit, The Second Affiliated Hospital and Yuying Children's Hospital of Wenzhou Medical University, Wenzhou, China

"These authors contributed equally to this work.

Correspondence to: Yuanrong Dai. 109 Xueyuan West Road, Wenzhou, China. Email: daiyr@126.com; Songmin Ying. Yuhangtang Road No. 388, Hangzhou, China. Email: yings@zju.edu.cn; Xian Shen. 109 Xueyuan West Road, Wenzhou, China. Email: 18817350420@126.com.
\end{abstract}

Submitted May 08, 2020. Accepted for publication Aug 21, 2020.

doi: $10.21037 /$ jtd-20-1861

View this article at: http://dx.doi.org/10.21037/jtd-20-1861

Coronavirus disease 2019 (COVID-19) is caused by severe acute respiratory syndrome coronavirus 2 (SARS-CoV-2) (1). Given its rapid spread worldwide, the epidemiological and clinical features of COVID-19 mandate further clarification. According to previous Wuhan-based studies, which respectively included 41 (2), 99 (3) and 52 (4) laboratory-confirmed COVID-19 patients, SARS-CoV-2 mimicked severe acute respiratory syndrome coronavirus in terms of severity and high mortality, however, a subsequent study conducted on 13-cases in Beijing demonstrated that all patients were mildly infected (5). Results of these studies varied greatly and were contradictory, therefore, we collected the data from 64 patients admitted to Oujiangkou Branch, the Second Affiliated Hospital and Yuying Children's Hospital of Wenzhou Medical University, aiming to describe the epidemiological and clinical characteristics of COVID-19 patients in Wenzhou city which, although far away from Hubei Province, is the most-affected area in China outside Hubei.

For this retrospective study, 64 laboratory-confirmed COVID-19 patients, admitted from Feb 6, to Feb 11, 2020 in above mentioned hospital were recruited. Confirmation of SARS-CoV-2 was done by real-time RT-PCR assay of sputum, throat-swab specimen. Patients were categorized into 3 groups, uncomplicated illness, mild-pneumonia and sever-pneumonia respectively, according to the severity defined by World Health Organization interim guidance (6). Epidemiological, demographic, clinical, laboratory and radiological data were extracted from medical records of patients. CT score was obtained by synthesizing the grading of range and density of the exudative lesion, following protocol from a previous study (7). Present study was approved by the Ethics Committee of above-mentioned Hospitals of Wenzhou Medical University (L-2020-13), along with a waiver of informed consent.

Of all the 64 cases, 38 (59.4\%) were male. Median age was 48.5 years (range, $10-77)$. Fever $(71.9 \%)$ and cough $(64.1 \%)$ were the most common symptoms, whereas nausea/vomiting (3.1\%), abdominal pain/diarrhea (10.9\%), and conjunctival congestion (1.6\%) were rare (Table 1). A 5 th-generation case was found in the study (Figure 1A). Patient 1 (Generation 2), who had never contacted with wildlife or Huanan Seafood Market, transmitted the SARSCoV-2 to Patient 2 (Generation 3) during his visit. The latter transmitted the virus to his family members (Patient 3 \& 4, Generation 4). Finally, the virus reached to hairstylist (Patient 5, Generation 5) when he repeatedly contacted with one of the family members and got laboratory-confirmed 
Table 1 Clinical features of patients with COVID-19

\begin{tabular}{|c|c|c|c|c|c|c|c|}
\hline Clinical features & $\begin{array}{l}\text { All patients } \\
\quad(n=64)\end{array}$ & $\begin{array}{l}\text { Uncomp. } \\
\qquad(n=3)\end{array}$ & Mild $(n=57)$ & Severe $(n=4)$ & $\begin{array}{l}\text { P value (mild } \\
\text { vs. uncomp.) }\end{array}$ & $\begin{array}{c}\text { P value (severe } \\
\text { vs. uncomp.) }\end{array}$ & $\begin{array}{c}\text { P value (severe } \\
\text { vs. mild }\end{array}$ \\
\hline Age (years), median [range] & $48.5[10-77]$ & 19 [10-32] & $49[20-77]$ & $44[41-49]$ & - & - & 0.11 \\
\hline Family onset, (\%) & 25/64 (39.1) & - & - & - & - & - & - \\
\hline \multicolumn{8}{|l|}{ Symptoms, (\%) } \\
\hline Fever & 46/64 (71.9) & $1 / 3(33.3)$ & $41 / 57$ (71.9) & 4/4 (100.0) & 0.21 & 0.14 & 0.57 \\
\hline Cough & $41 / 64(64.1)$ & 0/3 (0.0) & $39 / 57(68.4)$ & $2 / 4(50.0)$ & 0.039 & 0.43 & 0.59 \\
\hline Sputum production & 23/64 (35.9) & $0 / 3(0.0)$ & $21 / 57$ (36.8) & $2 / 4(50.0)$ & 0.55 & 0.43 & 0.63 \\
\hline Myalgia & $15 / 64(23.4)$ & 1/3 (33.3) & $12 / 57(21.1)$ & $2 / 4(50.0)$ & 0.53 & - & 0.22 \\
\hline Nausea/vomiting & $2 / 64(3.1)$ & $0 / 3(0.0)$ & $2 / 57(3.5)$ & $0 / 4(0.0)$ & - & - & - \\
\hline \multicolumn{8}{|l|}{ Laboratory findings } \\
\hline \multicolumn{8}{|l|}{ White blood cell $\left(\times 10^{9} / \mathrm{L}\right)$} \\
\hline Mean (SD) & $4.77(1.23)$ & $5.70(2.21)$ & $4.67(0.21)$ & $5.49(0.60)$ & 0.16 & 0.86 & 0.18 \\
\hline$<4,(\%)$ & $16 / 64(25.0)$ & 1/3 (33.3) & $15 / 57(26.3)$ & $0 / 4(0.0)$ & - & 0.43 & 0.56 \\
\hline \multicolumn{8}{|l|}{ Lymphocyte (×10 /L) } \\
\hline Mean (SD) & $1.36(0.44)$ & $1.86(0.76)$ & $1,35(0.41)$ & $1.09(0.39)$ & 0.51 & 0.14 & 0.22 \\
\hline$<1.1,(\%)$ & 17/64 (26.6) & $0 / 3(0.0)$ & 15/57 (26.3) & $2 / 4(50.0)$ & 0.57 & 0.43 & 0.31 \\
\hline \multicolumn{8}{|l|}{ Eosinophil (×10\% /L) } \\
\hline Mean (SD) & $0.055(0.062)$ & $0.063(0.051)$ & $0.057(0.064)$ & $0.028(0.045)$ & 0.87 & 0.38 & 0.37 \\
\hline \multicolumn{8}{|l|}{$\mathrm{CRP}(\mathrm{mg} / \mathrm{L})$} \\
\hline \multicolumn{8}{|l|}{ CT findings } \\
\hline Left lung involvement only, (\%) & $4 / 64(6.3)$ & $0 / 3(0.0)$ & $4 / 57(7.0)$ & $0 / 4(0.0)$ & - & - & - \\
\hline Right lung involvement only, (\%) & $7 / 64$ (10.9) & $0 / 3(0.0)$ & $7 / 57$ (12.3) & $0 / 4(0.0)$ & - & - & - \\
\hline Bilateral involvement, (\%) & $50 / 64(78.1)$ & $0 / 3(0.0)$ & $46 / 57(80.7)$ & 4/4 (100.0) & 0.011 & 0.029 & - \\
\hline CT score, mean (SD) & $24.9(22.2)$ & $0(0.0)$ & $22.2(16.24)$ & $47.8(2.90)$ & - & - & 0.0028 \\
\hline \multicolumn{8}{|l|}{ Medical therapies, (\%) } \\
\hline Interferon & 49/64 (76.6) & $3 / 3(100.0)$ & 45/57 (78.9) & $1 / 4(25.0)$ & - & 0.14 & 0.043 \\
\hline Antiviral agents & $58 / 64(90.6)$ & 2/3 (66.7) & $53 / 57(93.0)$ & $3 / 4(75.0)$ & 0.23 & - & 0.30 \\
\hline Corticosteroid & 4/64 (6.3) & $0 / 3(0.0)$ & $1 / 57(1.8)$ & $3 / 4(75.0)$ & - & 0.14 & - \\
\hline Antibiotics & $11 / 64(17.2)$ & $0 / 3(0.0)$ & $9 / 57(15.8)$ & $2 / 4(50.0)$ & - & 0.43 & 0.15 \\
\hline Oxygen therapy, (\%) & 62/64 (96.9) & 3/3 (100.0) & $55 / 57(96.5)$ & 4/4 (100.0) & - & - & - \\
\hline Outcomes cure, (\%) & $64 / 64(100.0)$ & 3/3 (100.0) & $57 / 57(100.0)$ & 4/4 (100.0) & - & - & - \\
\hline
\end{tabular}

Data are presented as median (range), mean (standard deviation) or $n / N(\%)$, where $\mathrm{N}$ is the total number of patients. $\mathrm{P}$ values comparing uncomp., mild, and severe respectively are from $\chi^{2}$, Fisher's exact test, independent-samples $t$-test, or one-way ANOVA. Uncomp., uncomplicated illness; mild, mild pneumonia; severe, severe pneumonia. 

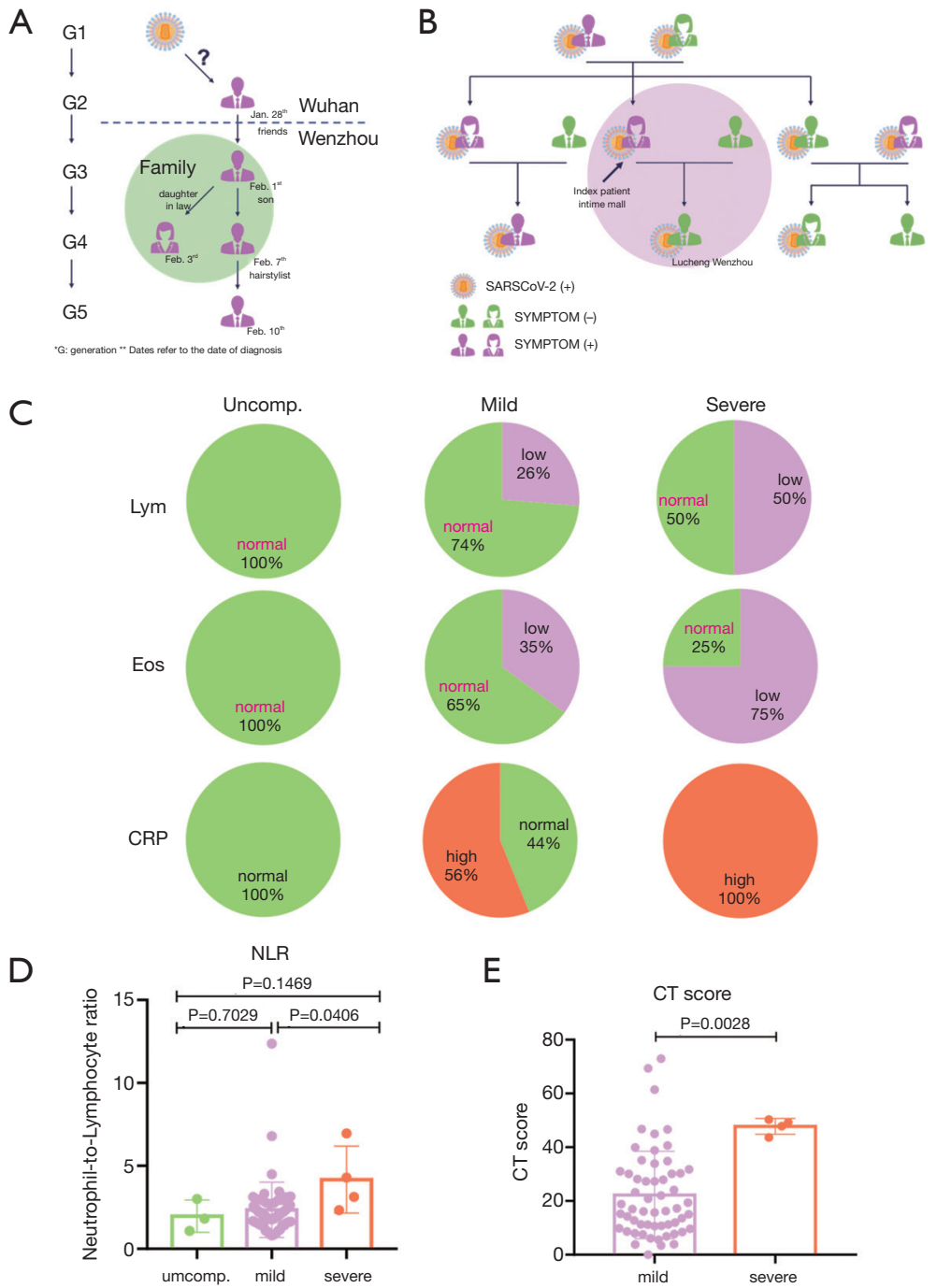

Figure 1 Demographic and clinical features of patients with COVID-19. (A) A 5th-generation case. P1 (G2), who had never contacted with wildlife or Huanan Seafood Market, came back from Wuhan and visited his friend (P2, G3) on Jan 19, the next day after which P1 (G2) developed fever and diagnosed COVID-19 On Jan 28, 2020. On Jan 31, 2020, 12 days after the visit, P2 was also diagnosed positive for COVID-19 on Feb 1 after having fever for 1-day. Later, P2's daughter-in-law (P3, G4) and son (P4, G4) also developed symptoms and were confirmed on Feb 3 and Feb 7, 2020, respectively. During this period, Patient 4 contacted his hairstylist (P5, G5) on Jan 29, and Jan 31, who developed fever on Feb 9 and then finally was laboratory-confirmed for COVID-19 on Feb 10, 2020. (B) 9-member cluster from a 12-member family, originated from a local epidemic area (Intime Mall). Index patient in this family cluster worked at Intime Mall and lived with her husband and son in Lucheng District. The couple and their son traveled to their hometown in Wencheng District on Jan 23, 2020, and repeatedly visited all of their relatives after that. 8 days later, the index patient's father developed fever and diagnosed COVID-19 on Feb 2, 2020. One of her sister-in-law developed fever on Feb 3, 2020, and got diagnosed with COVID-19 the following day. The index patient felt no symptoms until she finally developed fever on Feb 5, 2020 and then laboratory-confirmed COVID-19, on the same day. Afterwards, all of the remaining 9 members of the family underwent SARS-CoV-2 RNA detection and 6 were confirmed positive, among whom 4 were asymptomatic. Although 2 patients of this family cluster were admitted in another hospital, we obtained their information by the detailed description of their relatives in our hospital. *, G: generation; **, dates refer to the date of diagnosis. Patients were grouped into three categories as per WHO interim guidance. Uncomp., uncomplicated illness; mild, mild pneumonia; severe, severe pneumonia. (C) Patients with abnormal lymphocyte count, eosinophil count and CRP of different groups were analyzed. (D) Neutrophil-to-lymphocyte ratio was compared among three groups. (E) Comparison of CT score was made among two groups with pneumonia. 
for COVID-19 on the following day.

Twenty-five (39.1\%) cases were involved in SARSCoV-2 infected family clusters (Table 1), among them was a 9 -member cluster from a 12 -member family, which originated from a local epidemic area (Intime-Mall). Index patient worked at a local epidemic Mall. She spread the virus to 8 other members of the family when she traveled to her hometown in another District with her husband and son (Figure 1B). Presence of a considerable number of family clusters strengthened.

Lymphopenia was observed in 15 (26.3\%) patients with mild-pneumonia and $2(50.0 \%)$ patients with severepneumonia. Eosinophils reduction was also documented in $20(35.1 \%)$ patients of the mild-pneumonia group and $3(75.0 \%)$ of severe-pneumonia group. CRP rose in 32 (56.1\%) patients of mild-pneumonia group and $4(100 \%)$ of severe-pneumonia group, but in none of the uncomplicatedillness group (Table 1, Figure 1C). Neutrophil to lymphocytes ratio (NLR) of severe-pneumonia group was significantly higher than that of mild-pneumonia group $(\mathrm{P}=0.0406)$ (Figure 1D). Level of D-Dimer rose in $20(36.4 \%)$ of 55 patients of mild-pneumonia group and $3(75 \%)$ of 4 patients of severe-pneumonia group while normal levels of alanine aminotransferase, PCT, bilirubin, serum creatinine were documented only in a small number of patients (data not shown). CT findings showed that 50 (78.1\%) patients manifested bilateral involvement. Average CT score was $24.9 \pm 22.2$. Compared to that of mild-pneumonia group $(22.2 \pm 16.24)$, CT score of the severe-pneumonia group (47.8 \pm 2.9$)$ was markedly higher $(\mathrm{P}=0.0028)$ (Table 1, Figure $1 E)$. All 64 patients in our study were cured and discharged till March 10, 2020, although 3 of them were transferred to Intensive Care Unit once during treatment. In this study, the cure rate was $100 \%$ and the mortality was 0 (Table 1).

For treatment, oxygen therapy, antiviral therapy, interferon inhalation, intravenous antibiotics and glucocorticoid therapy were selectively initiated in different patients (Table 1).

Before the official announcement of Wuhan lockdown, around 20,000 Wenzhou businessmen traveled back to Wenzhou for the spring festival, thus making it the most seriously hit city outside Hubei, where then strictest quarantine strategy was implemented. Interestingly, we found that the severity and mortality in Wenzhoucases were much milder than those in Hubei-cases. A significantly lower fatality rate was found in Wenzhou (outside Hubei), compared to previous Wuhan-based studies where it was $15 \%$ (2) and $4.3 \%$ (8). Moreover, we discovered that rate of severity and complications related to COVID-19 was very low in Wenzhou, as compared to those in Wuhan. The low mortality in Zhejiang Province (9) suggests the prominent role of quarantine policies and emphasizes their strict implementation as the global epidemic continues to worsen (10).

Family clusters accounted for $39.1 \%$ of all cases in our study, strengthening the idea of efficient human-to-human transmission. Consistently, strong longitudinal infectivity was further proved by our discovery of a 5 th-generation COVID-19 case. Our study also revealed the presence of asymptomatic cases $(9.4 \%)$, which might be a potential source of infection. Aiming to extend the diagnostic parameters, we looked for new markers to predict the severity of COVID-19 and discovered that NLR and CT score were significantly elevated in severe-pneumonia group.

Presence of a considerable number of asymptomatic patients in our study cohort draws attention towards more strict screening and contact tracing of infected individuals, because of the likelihood that presence of asymptomatic patients might lead to misdiagnosis of the potential infected source and contribute to the rapid spread of the virus. Specifically involving Wenzhou-patients, the study is limited by the small sample size of severe-pneumonia group. Finally, considering the recruitment of Wenzhou-based patients only, our study depicts a true picture of the clinical condition and characteristics of patients outside Hubei.

\section{Acknowledgments}

We acknowledge all the patients involved in our study and all the health-care workers involved in treatment and diagnosis of patients in Wenzhou.

Funding: This study was supported by National Natural Science Foundation of China (grant numbers 81800028 , 81570027).

\section{Footnote}

Provenance and Peer Review: This article was a free submission to the journal. The article was sent for external peer review.

Conflicts of Interest: All authors have completed the ICMJE uniform disclosure form (available at http://dx.doi. org/10.21037/jtd-20-1861). The authors have no conflicts of interest to declare. 
Ethical Statement: The authors are accountable for all aspects of the work (including full data access, integrity of the data and the accuracy of the data analysis) in ensuring that questions related to the accuracy or integrity of any part of the work are appropriately investigated and resolved.

Open Access Statement: This is an Open Access article distributed in accordance with the Creative Commons Attribution-NonCommercial-NoDerivs 4.0 International License (CC BY-NC-ND 4.0), which permits the noncommercial replication and distribution of the article with the strict proviso that no changes or edits are made and the original work is properly cited (including links to both the formal publication through the relevant DOI and the license). See: https://creativecommons.org/licenses/by-nc-nd/4.0/.

\section{References}

1. Chen L, Liu W, Zhang Q, et al. RNA based mNGS approach identifies a novel human coronavirus from two individual pneumonia cases in 2019 Wuhan outbreak. Emerg Microbes Infect 2020;9:313-9.

2. Huang C, Wang Y, Li X, et al. Clinical features of patients infected with 2019 novel coronavirus in Wuhan, China. Lancet. 2020;395:497-506.

3. Chen N, Zhou M, Dong X, et al. Epidemiological and clinical characteristics of 99 cases of 2019 novel coronavirus pneumonia in Wuhan, China: a descriptive study. Lancet 2020;395:507-13.

4. Yang X, Yu Y, Xu J, et al. Clinical course and outcomes

Cite this article as: Qiu Z, Dai W, Syeda MZ, Ouyang L, Lin J, Sun L, Leng Z, Chen X, Xu X, Zhao Y, Ying B, Shen X, Ying S, Dai Y. Clinical features of 64 patients (outside Hubei) with COVID-19 in Wenzhou, China. J Thorac Dis 2020;12(10):61276131. doi: $10.21037 /$ jtd-20-1861 of critically ill patients with SARS-CoV-2 pneumonia in Wuhan, China: a single-centered, retrospective, observational study. Lancet Respir Med 2020;8:475-81.

5. Chang D, Lin M, Wei L, et al. Epidemiologic and Clinical Characteristics of Novel Coronavirus Infections Involving 13 Patients Outside Wuhan, China. JAMA 2020;323:1092-3.

6. WHO. Clinical management of severe acute respiratory infection when novel coronavirus $(2019-\mathrm{nCoV})$ infection is suspected. 2020. Available online: https://apps.who.int/iris/ handle/10665/331446? search-result=true \&query $=10665 \%$ $2 \mathrm{~F} 331446 \&$ scope $=\& \mathrm{rpp}=10 \&$ sort $\_$by $=$score $\&$ order $=$ desc

7. Xie H, Huang X, Zhong X, et al. Pneumocystis jiroveci pneumonia in AIDS: the correlation between SLDH and CT quantitive score of exudative lesion. Electron J Emerg Infect Dis 2018;3:15-20.

8. Wang D, Hu B, Hu C, et al. Clinical Characteristics of 138 Hospitalized Patients With 2019 Novel CoronavirusInfected Pneumonia in Wuhan, China. JAMA 2020;323:1061-9.

9. $\mathrm{Xu} X, \mathrm{Wu} X$, Jiang $\mathrm{X}$, et al. Clinical findings in a group of patients infected with the 2019 novel coronavirus (SARSCov-2) outside of Wuhan, China: retrospective case series. BMJ 2020;368:m792.

10. Aggarwal S, Garcia-Telles N, Aggarwal G, et al. Clinical features, laboratory characteristics, and outcomes of patients hospitalized with coronavirus disease 2019 (COVID-19): Early report from the United States. Diagnosis (Berl) 2020;7:91-6. 